\title{
In Vivo Photoacoustic Tracking of Mesenchymal Stem Cell Viability
}

Kabir S. Dhada, Derek S. Hernandez, Laura J. Suggs

Department of Biomedical Engineering, University of Texas at Austin, Austin, Texas 78712

Corresponding Author: Laura Suggs, suggs@utexas.edu

SUPPLEMENTARY FIGURES 


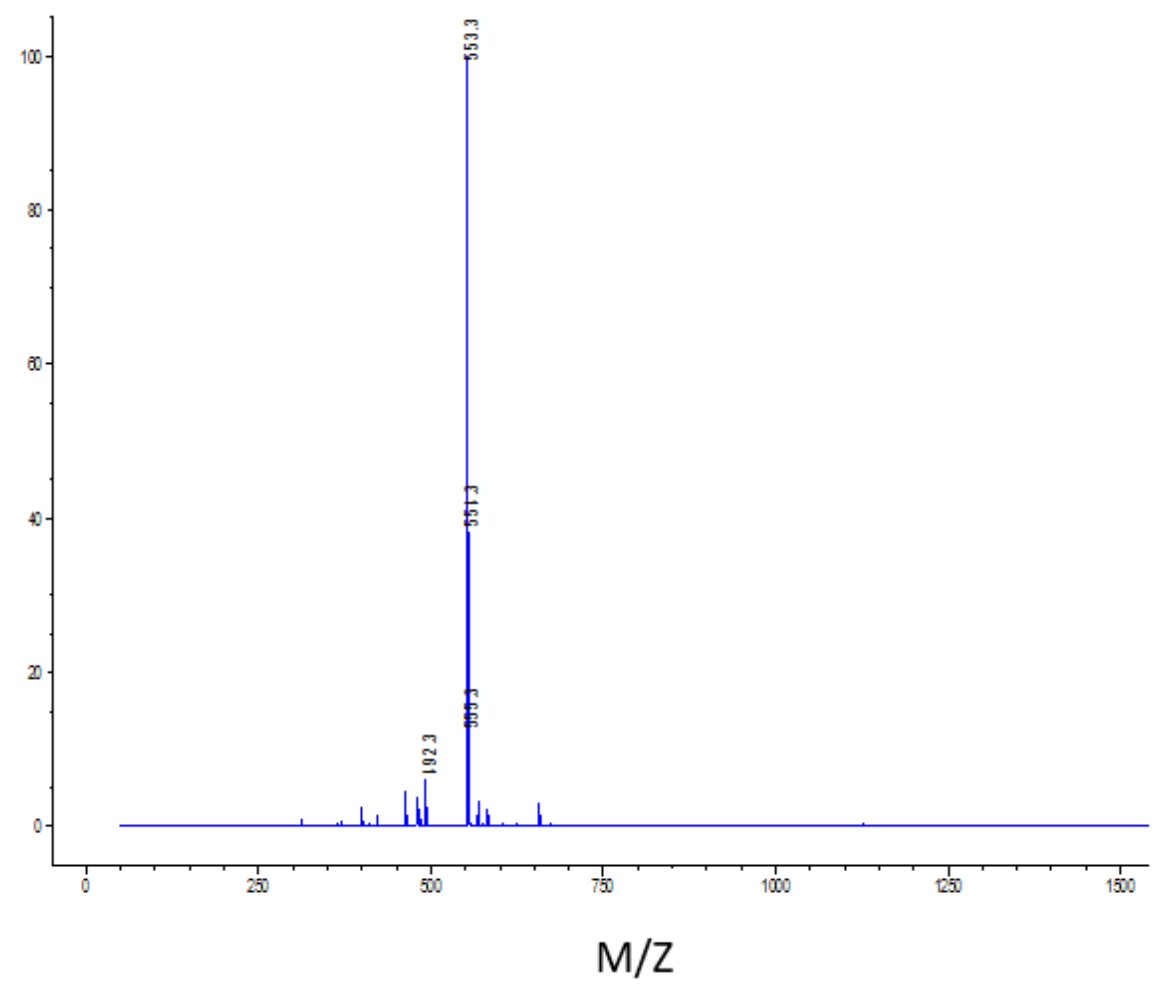

Supplementary Figure S1. Mass spectroscopy displays peak of $553.3 \mathrm{~m} / \mathrm{z}$ for modified IR775 into IR775C.

A

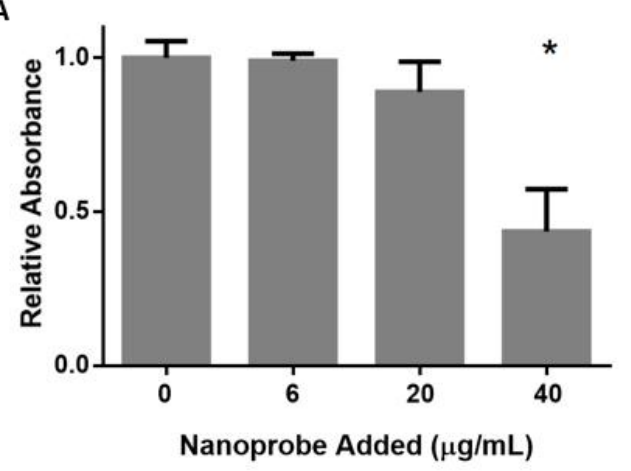

B

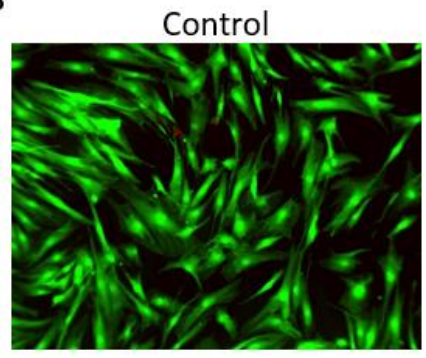

Nanoprobe

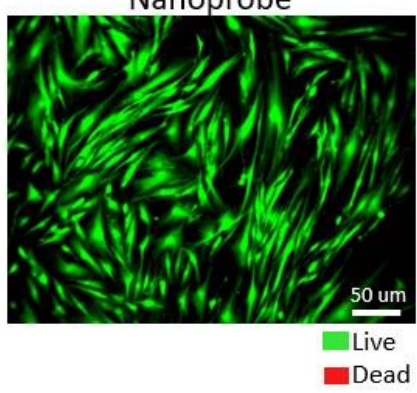

Supplementary Figure S2. Nanoprobe effect on MSCs. (A) MTS assay displaying effect of nanoprobe on MSCs. Increase in concentration of nanoprobe has little effect on viability until $40 \mu \mathrm{g} / \mathrm{mL}$. (B) Live/Dead assay displays no noticeable difference in viability and morphology of MSCs incubated with $20 \mu \mathrm{g} / \mathrm{mL}$ nanoprobe compared to control group. 
A

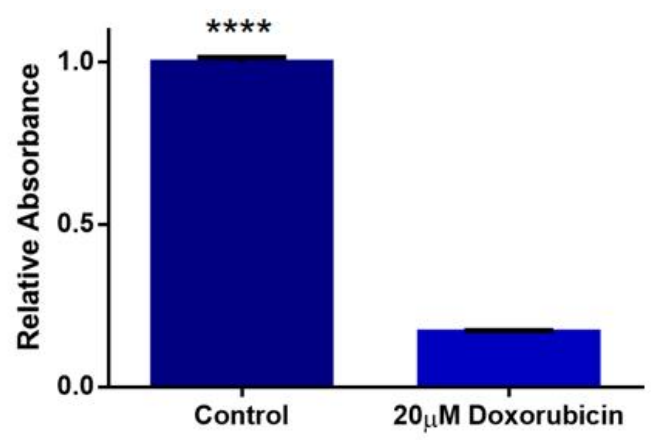

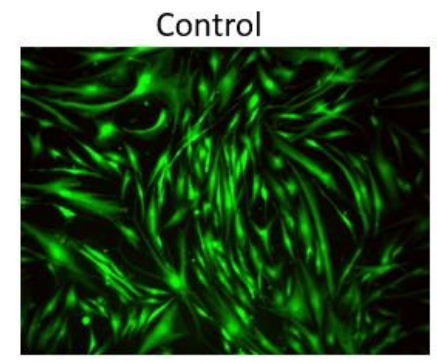

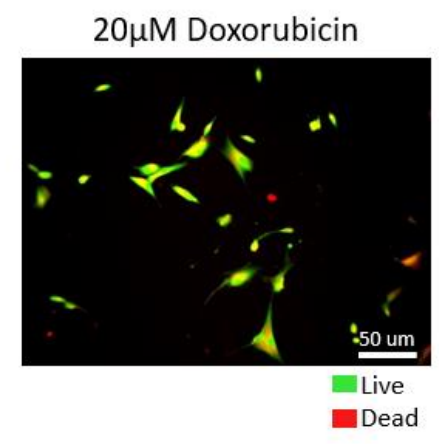

Supplementary Figure S3. Effect on MSCs by incubating with $20 \mu \mathrm{M}$ Doxorubicin for 48 hours. (A) MTS assay shows doxorubicin reduced MSC viability down to $17 \%$ compared to control group. (B) Live/Dead assay displays reduced number of MSCs and overlap of live and dead stain on all MSCs within field of view due to cell death. $* * * * p<0.0001$

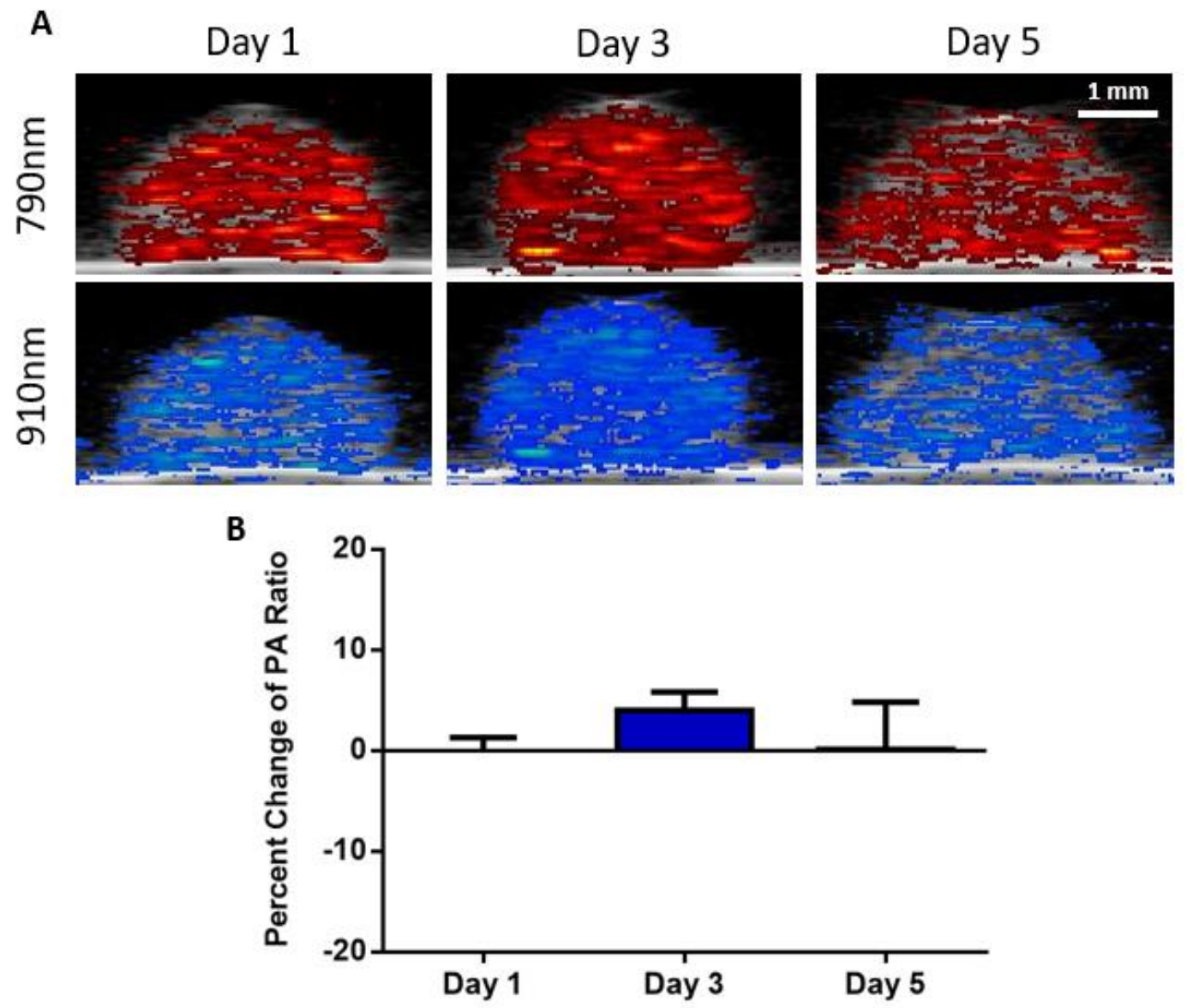

Supplementary Figure S4. Longitudinal study of nanoprobe loaded MSCs. (A) MSCs were imaged on days 1, 3, and 5 after loading to determine stability of nanoprobe in viable cells. (B) Percent change of 790nm/910nm ratio displayed no significant changes over 5 days. 

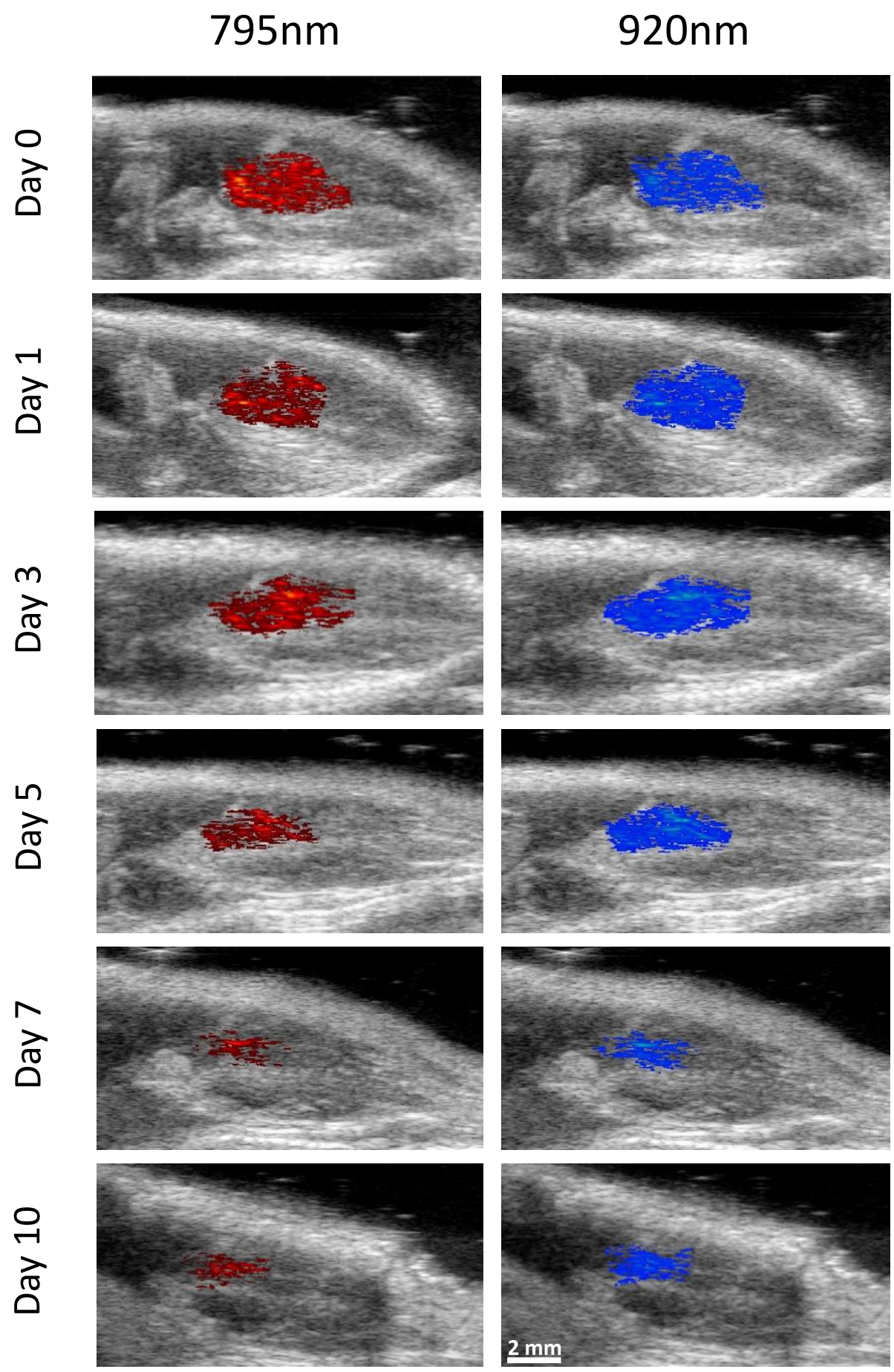

Supplementary Figure S5. US/PA images of injected MSCs at $795 \mathrm{~nm}$ and $920 \mathrm{~nm}$. Representative images at $795 \mathrm{~nm}$ and $920 \mathrm{~nm}$ of MSCs at each time point are displayed. These images were used for creating the ratiometric images in Figure 7. 